Ann. Biol. anim. Bioch. Biophys., I96r, 1 (4), 449-456.

\title{
APPLICATION DE LA MÉTHODE DE NESSLER AU MICRODOSAGE DE L'AZOTE ALPHA-AMINÉ EN MILIEU BIOLOGIQUE
}

\author{
M. C. MICHLL \\ Aves la collaboration technique de Simone Bocne \\ Service de Biochimie et de Nutrition, \\ Centre national de Recherches zootechniques, Jouy-en-Josas (Seine-et-Oise)
}

\section{SOMMAIRE}

$1^{\circ}$ Lal réaction de Nessler, pour le dosage de cuantités d'azote ammoniacal de l'ordre de ro $\gamma / \mathrm{ml}$, a été étudiée quant à la préparation du réactif et la formation du dérivé coloré (influence de la température, de la dilution, etc.)

2" Les acides aminés, dégradés far la ninhydrine en milieu sulfurique, foumissent de l'azote anmmoniacal (ui est dosé après microdiffusion. Les résultats obtenus sont voisins de la valeur théorique, sauf pour la proline (39 p. 10o), le tryptophane ( 53 P. Ioo) et l'acide aspartique ( 88,6 p. I00). La cystine fournit des résultats aberrants.

i" Le groupement amidé de l'asparagine et de la glutamine n'est pas dégradé dansces conditions.

$t^{\prime \prime}$ Jes peptides fournissent des valeurs faibles (de 10 ì $30 \mathrm{p}$. 100).

$\sigma^{-3}$ Cette méthode a été comparée, sur des mélanges de produits purs et des extraits de sang, à la méthode de dosigre potentiométrique.

Dans une étude antérieure, nous avons décrit une méthode de dosage de l'azote aminé dans les milieux biologiques par dégradation à la ninhydrine et mesure titrinétrique, après nicrodiffusion, de l'ammoniac formé. Ce procédé est plus spécifique que celui de VAN Styke à l'acide nitreux, en particulier à l'égard des amides et de certains peptides (Micher, I960). 'loutefois ces deux méthodes offrent l'inconvénient de manquer de sensibilité : certains produits biologiques, tels que le sang, contiennent peu d'acides aminés libres ; en outre, on ne peut en prélever que de faibles quantités sur l'animal vivant pour éviter de perturber son métabolisme.

La technique que nous décrivons a pour but d'améliorer à la fois la spécificité et la sensibilité de la réaction à la ninhydrine. Elle a été comparée, sur des substances pures et des extraits de produits biologiques, à la méthode antérieurement décrite et à un procédé de dosage potentiométrique (VIGNERON I960). Ce demier permet de doser l'ensemble des groupements aminés libres sans dégradation des substances labiles. I e principe de chacune de ces méthodes est le suivant :

Io Méthode à la ninhydrine. - Par ébullition en milieu sulfurique dilué, et en 
présence de ninhydrine, le groupement alpha-aminé est dégradé. L'ammoniac formé, obtenu en solution sulfurique par microdiffusion, est dosé colorimétriquement par le réactif de NESSLER.

$2^{\circ}$ Méthode potentiométrique. - La solution à doser est débarrassée des sels minéraux par passage sur résine échangeuse d'ions. L'éluat séché est solubilisé dans l'acide acétique anhydre. Dans ces conditions, le groupement aminé se comporte comme une base quantitativement titrable par l'acide perchlorique.

\section{MATÉRIEI, ET MÉTHODES}

a) Riactifs

$\mathrm{H}_{2} \mathrm{SO}_{4} \mathrm{~N} / \mathrm{IOO}$

Perhydrol 100 volumes

Ninhydrine (solution à I p. roo dans $\mathrm{H}_{2} \mathrm{SO}_{4} \mathrm{~N} / 100$ )

Tampon de pH 6,6

$\begin{cases}\mathrm{H}_{2} \mathrm{PO}_{4} \mathrm{~K} & 3 \mathrm{Ig} \\ \mathrm{H}_{2} \mathrm{PO} \mathrm{K}_{2} & 27 \mathrm{~g} \\ \mathrm{H}_{2} \mathrm{O} \mathrm{O} . \mathrm{s} .1000 \mathrm{ml} & \end{cases}$

\section{Réactif de Nessler}

Dans $200 \mathrm{ml}$ de NaOII à 20 p. 100 (I'oids/volume), on ajoute successiventent, sous agitation : $\mathrm{Hg} \mathrm{I}_{2} 20 \mathrm{~g}$

$\mathrm{KI} I 3 \mathrm{~g}$

On ajoute à cette solution de l'iode, environ $2 \mathrm{~g}$, juscu à réaction positive à l'amidon. Elle est placée dans un flacon jaune, laissée 2 jours à température ambiante, puis décantée.

Avant le dosage, on dilue cette solution à raison de $3 \mathrm{p}$. 100, dans $H_{2}, 50 / \mathrm{N} / 100$ refroidi à $t_{5}^{-10}$. Le réactif dilué est placé au bain-marie à $35^{\circ}$.

$\mathrm{HClO}_{4} \mathrm{~N} / 10$ (préparé d'après VIGNERON I960),

Acide acétique R. P.,

Acide formique pour analyse "Prolabo ":

\section{b) Matériel}

Tubes de I $4 / 140 \mathrm{~mm}$ jaugés à 5 et $10 \mathrm{ml}$;

Tubes de $\mathrm{i} 6 / \mathrm{r} 60 \mathrm{~mm}$ calibrés au même diamètre.

Photomètre ERAL, muni d'un filtre interférentiel de longueur d'onde $440 \mathrm{mu}$.

PH mètre Radiometer 'Type 22 - équipé d'électrodes à distance type $202 \mathrm{~K}$;

Agitateur magnétique ;

Seringue automatique;

Bain-marie réglé à $35^{\circ} \mathrm{C} \pm 0,1$.

\section{c) Extraction des acides aminés à partir du sang}

Dès le prélèvement, $5 \mathrm{ml}$ de sang sont dispersés dans $75 \mathrm{ml}$ d'alcool à $85^{\circ}$. Après I heure à température ambiante, on filtre sur verre fritté à l'aide de supercel. Le résidu est rincé par $50 \mathrm{ml}$ d'alcool à $80^{\circ}$. Le filtrat est ensuite évaporé sous un courant d'air. Après séchage on reprend le résidu par $10 \mathrm{ml}$ de la solution de ninhydrine.

On effectue la dégradation dans des tubes jaugés de $1_{4} \times 140 \mathrm{~mm}$, contenant $4 \mathrm{ml}$ de solution (en double exemplaire). Après I 5 minutes à $100^{\circ} \mathrm{C}$, on ajoute $0,5 \mathrm{ml}$ d'eau oxygénée et porte de nouveau 5 minutes au bain-marie. Après refroidissement, le volume est amené à $5 \mathrm{ml}$ avec le tampon concentré. On effectue la micro-diffusion dans des cellules de CONWAY, avec I ml de solution (en double exemplaire), le compartiment intérieur contient $2,5 \mathrm{ml}$ de $\mathrm{Il}_{2} \mathrm{SO}_{4} \mathrm{~N} / \mathrm{roo}$. On déplace par I $\mathrm{ml}$ de solution de $\mathrm{K}_{2} \mathrm{Co}_{3}$, pendant 2 heures, puis on préleve deux fois I $\mathrm{ml}$ dans le compartiment intérieur. Cette solution contenue dans des tubes calibrés de $16 \times 160 \mathrm{~mm}$, est placée au bainmarie.

La réaction colorée s'effectue en injectant $5 \mathrm{ml}$ de réactif dilué par tube. Après agitation. on laisse développer ${ }_{5}$ minutes au bain-marie. La densité optíne est lue à 440 mu. sur un témoin $\mathrm{I}_{2} \mathrm{O}$ distillée, ceci afin de contrôler la valeur des réactifs. 


\section{d) Dosage potentiométrique}

L'échantillon à doser, contenant environ $\mathrm{r}$ mg d'azote aminé, est débarrassé des impuretés ca. tioniques par passage sur Permutite $\mathrm{P}$ 5o sous forme hydrogène. L'éluat, $\left(\mathrm{NH}_{4} \mathrm{OIl},{ }_{4} \mathrm{~N}\right)$ séché sous vide, est repris par $2 \mathrm{ml}$ d'acide formique, auquel on ajoute $40 \mathrm{ml}$ d'acide acéticque. On dose d'après Vigneron ( 1960 ). Le titrage est effectué à l'aide d une burette de $2 \mathrm{ml}$ au $\mathrm{I} / \mathrm{I} 00$, dont la pointe plonge dans le liquide. Au voisinage de la zone de virage, les quantités d'acide ajoutées sont de $25 \mu l$.

\section{RÉSULTATS OBTENUS}

$\mathrm{I}^{\circ}$ Mesure colorimétrique de $\left(\mathrm{NH}_{4}\right)^{\vdash}$ : étude de la réaction de Nessler.

Dans les conditions indiquées, avec différentes concentrations de $\left(\mathrm{NH}_{4}\right)_{2} \mathrm{SO}_{4}$ en solution sulfurique, la densité optique obtenue est la suivante : ('Tableau I et fig. I).

TABI,EAU I

\begin{tabular}{|c|c|c|}
\hline Sen ypar tube $(\mathrm{cml})$ & 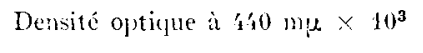 & Moyenme \\
\hline 0 & $11-12-10-12$ & 11,66 \\
\hline $\begin{array}{lll}-\cdot--\cdots & - \\
5 & & \end{array}$ & $117 \quad 121 \cdots 10 k-107$ & 113,25 \\
\hline $10-\cdots$ & $233 \geq 29-216-15$ & $2-2,5$ \\
\hline 15 & 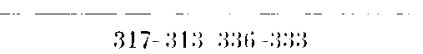 & 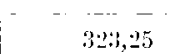 \\
\hline
\end{tabular}

La coloration se dévaloppe en Io minutes environ et reste stable pendant plusieurs heures. L'addition de réactif plus concentré (de 3 à 5 p. 100), n'a pas d'effet sur la valeur maximum de la coloration, mais on pent observer un louche avec les

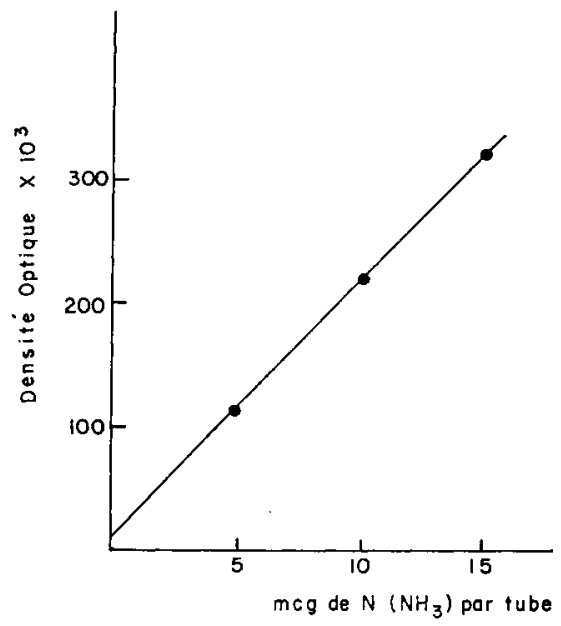

Fic. I. - Dosage colorimétrique divect.

fortes concentrations. En revanche, la température des solutions joue un rôle impor tant aux différents stades du dosage. La préparation du réactif dilué doit être effectuée à une température de $1^{\prime \prime}$ ordre de $15^{\circ} \mathrm{C}$, ceci afin de diminuer la valeur du blanc. Cette action s'exerce aussi au cours de la réaction colorée : le maximum obtenu est fonction de la température (fig. 2). 
I,es valeurs obtenues après microdiffusion sont égales ou légèrement supérieures à celles du dosage direct, vraisemblablement à cause de contaminations extérieures. Pour annuler cette cause d'erreur, une gamme étalon, obtenue après diffusion, est préparée pour chaque série de dosages (fig. 3). A $20^{\circ} \mathrm{C}$ la diffusion requiert environ $2 \mathrm{~h}$ pour être quantitative (fig. 4). Enfin la reproductibilité des résultats, obtenus par diffusion de solutions à Io et $20 \% / \mathrm{ml}$ d'azote ammoniacal, est reportée dans le tableau II.

TABL,EAL 2

Coefficient de wariation mesur' sur ti essais suctessifs dans chaque série.

\begin{tabular}{c|c|c}
\hline & Série 1 & Série 2 \\
\hline $10 \% / \mathrm{ml}$ & $-0.3 \mathrm{p} \cdot 100$ & $1,0 \mathrm{p} \cdot 100$ \\
\hline $00 \mathrm{\gamma} / \mathrm{ml}$ & $0,75 \mathrm{pl} \cdot 100$ & $1,3 \mathrm{p} \cdot 100$ \\
\hline
\end{tabular}

$2^{\circ}$ Dégradation du groupement aminé par la ninhydrine et mesure de $\mathrm{NH}_{3}$ formé.

Le tableau 3 résume les résultats obtenus. Les substances à doser, à la concentration $\mathrm{M} / \mathrm{I}$ ooo, ont été traitées de la manière indiquée, le pourcentage de clégradation est exprimé par rapport à un grotipement aminé par molécule.

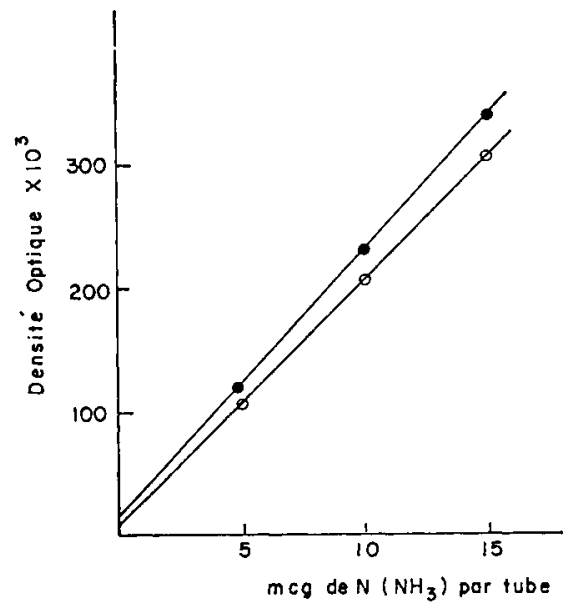

FIS. 2. - Inthuence de la temprírature sur le déreloppement de la coloration;

$$
\begin{aligned}
& -20^{\circ} \mathrm{C} \\
& -40^{\circ} \mathrm{C}
\end{aligned}
$$

$3^{\circ}$ Dosage de l'azote aminé du sang après extraction alcoolique. Comparaison arce un mélange d'acides aminés purs de concentration roisine.

Divers extraits alcooliques de sang, soit de porc, soit de poulet, ont été dosés : I), par la méthode antérieurement décrite, 2) par la présente méthode, et 3), par potentiométrie après passage sur résine. Les valeurs movennes (5 déterminations dans chaque cas), ont été comparées à celles obtenues avec un mélange d'acides 


\section{TABLEAU 3}

Dégradation de diverses substances par la ninhydrine:

Pourcentage d'ammoniac obtenu par rapport à un groupement aminé par molécule.

\begin{tabular}{|c|c|c|c|}
\hline Acides aminés & P. 100 obtenu & Amides et peptides & 1. 100 obtenu \\
\hline Glycine $\ldots \ldots \ldots \ldots \ldots \ldots$ & 99,9 & Glutamine ........... & 100,8 \\
\hline 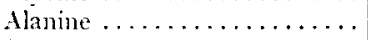 & 99,8 & Asparagine .............. & 101,0 \\
\hline Sérine $\ldots \ldots \ldots \ldots \ldots \ldots \ldots$ & 100,0 & Glycyl glycine............. & 31,1 \\
\hline Thróonine ............... & 99,6 & Glycyl-I-Leucine $\ldots \ldots \ldots \ldots$ & 30,5 \\
\hline Valine..$\ldots \ldots \ldots \ldots \ldots$ & 101,1 & Glycyl-L-Tyrosine . ........ & $\$ 5,1$ \\
\hline Jeucine $\ldots \ldots \ldots \ldots \ldots \ldots$ & 99,0 & Digrlycyl-glycine $\ldots \ldots \ldots \ldots$ & 3,6 \\
\hline Isoleucine $\ldots \ldots \ldots \ldots \ldots \ldots$ & 99,0 & Lencyl-Glycine . . . . . . . . . . & $5, ?$ \\
\hline Acide Glutamique...... . & 101,0 & Lencyl-glycyl-glycine ....... & 9,8 \\
\hline - Aspartique .......... & 88,6 & Carnosine $\ldots \ldots \ldots \ldots \ldots$ & 17,0 \\
\hline Arginine $\ldots \ldots \ldots \ldots \ldots$ & 99,5 & Ifistidyl-Iristidine . . . . . . . & 31,7 \\
\hline Lrsine $\ldots \ldots \ldots \ldots \ldots \ldots$ & 107,1 & Glutathion $\ldots \ldots \ldots \ldots \ldots$ & 105,5 \\
\hline Phénylalanine............ & 99,7 & & \\
\hline Tyrosine $\ldots \ldots \ldots \ldots \ldots \ldots$ & 98,0 & & \\
\hline Méthionine .............. & 100,1 & & \\
\hline IIistidine $\ldots \ldots \ldots \ldots \ldots$ & 110,5 & & \\
\hline Tryptophane............. & $5 \%$ & & \\
\hline Proline $\ldots \ldots \ldots \ldots \ldots \ldots$ & 39,1 & & \\
\hline Cystine $* \ldots \ldots \ldots \ldots \ldots$ & 132 & & \\
\hline Cystéine ${ }^{*} \ldots \ldots \ldots \ldots \ldots$ & 26 & & \\
\hline
\end{tabular}

aminés traité de la même manière. Ce mélange contient les acides aminés du tableau 3, plus la glutamine, à l'exclusion de l'acide aspartique, de la cystine et de la cystéine.

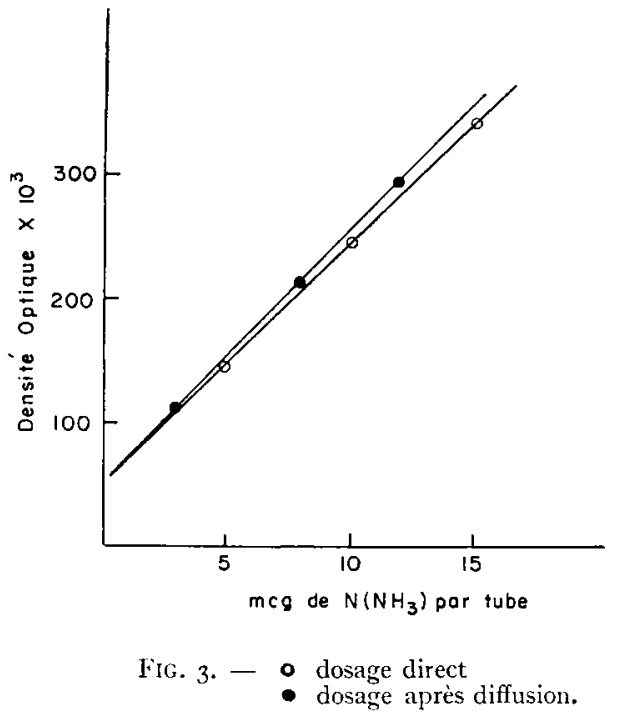

Ces substances sont diluées en proportions équimoléculaires, puis leur concentration en azote aminé est amenée à $7,85 \mathrm{mg}$ par Ioo $\mathrm{ml}$ (valeur intermédiaire entre celle du sang des oiseaux et des monogastriques). Les résultats obtenus sont reportés dans le tableau 4 . 


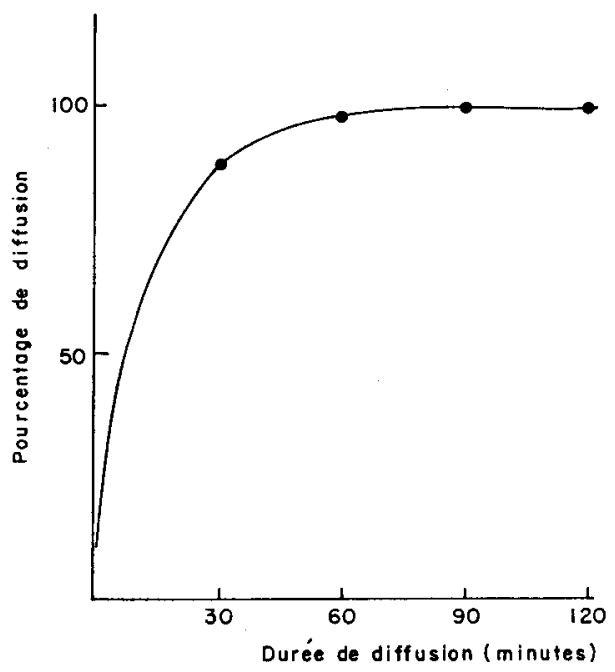

Fig. 4. - Vilesse de difusion $\mathrm{N}\left(\mathrm{NH}_{3}\right):$, $\mathrm{mog} / \mathrm{ml}$.

TABIEAU 4

Dosage de l'azote aminé du sang et d'un mélange standard (Valeur exprimées en mg d'azote aminé dans $100 \mathrm{ml}$ ). Méthode employée

\begin{tabular}{l|c|c|c}
\hline & Ninhydrine titrimétrie & Ninhydrine Nessler & Potentiométrie \\
\cline { 2 - 4 } & 7,6 & 7,5 & 8,7 \\
Mélange standard *... & 6,60 & 5,86 & 7,52 \\
Sang de porc ...... & 12,0 & 9,0 & $18, \%$ \\
Sang de poulet ..... & & \\
\hline
\end{tabular}

* Par rapport à la théorie, la valeur expérimentale attendue est de 97 p. 100 pour la ninhydrine. Eille est de 112 p. 100 pour la potentionétrie (2 fonctions aninées dosables pour l'arginine, la lysine et la cystine).

\section{DISCUSSION}

La réaction de NESSLER (I856), a été étudiée et utilisée par Folin (IgI2), pour le dosage de l'azote ammoniacal dans des produits biologiques. Elle a été appliquée ensuite à la détermination directe de faibles quantités d'azote organique, après minéralisation par la méthode de KJELdHAL. (Koch I924; Yuen et PoliLARd I952. ShaFFER et SPRECHER I957, IAANG I958; Burck I960; RAO I960). Les causes d'erreurs sont nombreuses. HETRICK et WHITNEy (I949) Thompson et MoRRIson (I95I), Herbain (I953) ont montré l'influence du $\mathrm{pH}$ de la réaction, de sa température et de divers cations sur la formation du complexe coloré. La présence d'un louche out d'un précipité est fréquemment rencontrée. Ceci est dû au fait que la réaction est fonction des proportions relatives des ions $(\mathrm{Hg} \mathrm{I} 4)^{-},(\mathrm{OH})^{-}$et $\left(\mathrm{NH}^{4}\right)^{+},($SARKAR et Grosh, I956).

L'obtention d'une coloration dont l'intensité est une fonction linéaire de la 
concentration en $\left(\mathrm{NH}^{4}\right)^{+}$dépend dans une large mesure de la quantité d'iode présente dans le réactif. En effet, les formules à teneur faible en cet élément (CoNwAy I957, BuRck I960) fournissent une courbe qui s'infléchit rapidement pour des taux de $\left(\mathrm{NH}^{4}\right)^{+}$supérieurs à $5 \mathrm{\gamma} / \mathrm{ml}$. L'inverse se produit avec des teneurs plus élevées en iode (RAO I960). Dans ce dernier cas, l'apparition d'un trouble est fréquente.

La méthode que nous proposons a pour but d'éliminer la plupart de ces causes d'erreurs. Les proportions relatives des différents réactifs, la température, sont exactement déterminées pour toute une série de dosage. I a microdiffusion de $\left(\mathrm{NH}^{4}\right)^{+}$ en solution sulfurique permet d'éliminer l'interférence possible des autres cations.

Cette méthode a été appliquée au dosage de $\left(\mathrm{NH}^{4}\right)^{+}$libéré par l'action de la ninhydrine sur les acides aminés. Les conditions de dégradation en milieu sulfurique dilué, puis de microdiffusion, ont été modifiées afin d'obtenir une réaction plus spécifique. Les résultats obtenus, pour la presque totalité des acides aminés, isolés ou en mélange, sont proches de la théorie. En revanche, les amides et peptides sont nettement moins dégradés que par la méthode antérieurement décrite et à fortiori par le procédé VAN SLYKE à l'acide nitreux. Ces différences se retrouvent dans le dosage de la fraction aminée du sang. La comparaison avec la méthode potentiométrique permet de mettre en évidence dans certains cas (sang de poulet), la présence d'une importante fraction peptidique; celle-ci est absente du sang du monogastrique, ainsi que l'ont montré STEIr et MOORE (I954).

En résumé, la méthode proposée permet de doser avec une spécificité et une précision satisfaisante des quantités d'azote aminé de l'ordre de Io $\gamma$ par $\mathrm{ml}$.

Reçu en juillet 1961.

\section{SUMMARY}

APPLICATION OF TIIE NESSIER METHOD TO THE ALPIA-AMINONITROGEN

MICRO-DETERMINATION IN BIOLOGICAL MEDIUM

$1^{o}$ The Nessler reaction for the determination of quantities of ammoniacal nitrogen for the bracket of $10 \mathrm{r} / \mathrm{ml}$ has been studied as to the preparation of the reagent and the formation of a colored derivative (influence of temperature, dilution etc.).

$2^{\circ} \quad$ The amino acids degraded by the ninhydrin in a sulfuric medium give ammoniacal nitrogen which is rated after microdiffusion. The results obtained are close to the theoretical values except for proline (30 p. 100), tryptophan (53 p. 100) and aspartic acid $(88,6$ p. 100). Cystine gives aberrant results.

$3^{\circ}$ The amide groups of asparagine and glutamine are not degraded under these conditions.

$4^{\circ}$ The peptides give low rations (from io to $3^{\circ} \mathrm{p}$. 100).

$5^{\circ}$ This method has been compared on mixtures of pure produces and on blood extracts with the potentiometric method of determination.

\section{RÉFÉRENCES BIBIIOGRAPHIQUES}

Bukck H. C. I96o. Kolorimetrische Mikro-Kjeldahl-Methode mit direkter Nesslerisation zur routinemässigen Stickstoffbestimmung (Nitro- und Nitrosogruppen ausgenommen). Mikrochem Acta., 200-203.

CoNway E. J., 1957. Microdiffusion analysis and volumetric error. 4th ed., Crosby Lockwood. London.

Folin O., FARuer G. J., igi 2. Total Nitrogen Determination. J. Biol. Chem., 11, 493.

HerbaIN M., I953. Sur l'adaptation analytique de la réaction de Nessler. Application aux milieux biologiques. I. Le dosage de 25 à $\gamma$ roo d'azote. Bull. Soc. Chim. Biol., 35, 1031-1039.

Hetrick J. H., WIIITNEY R. M., I949. Determination of nitrogen in milk by direct nesslerization of the digested sample. J. Dairy Sci., 32, I I t-I 22. 
Koch F. C., Mac Merkin 'T. L., I924. A new direct nesslerization micro Kjeldahl method and a modification of the Nessler - Folin reagent for ammonia. J. Amer. Chem. Soc., 46, 2066.

LANg C. A., r958. Simple microdetermination of Kjeldahl nitrogen in biological materials. Anal. Chem., 30, I $692-1694$.

Miciel M. C., ig6r. Dosage de l'azote aminé dans quelques liquides biologiques. Ann. Biol. Anim. Bioch. Biophys., 1, 248-255.

RAo M. B., Whitney R. M., I960. An Improvement in the Direct Nesslerization Method for the Determination of 'Total Nitrogen in Sto-Sterile Milk. J. Dairy Sci., 43, 563-565.

Sarkar P. B., Ghosh N. N., 1956. Studies on Nessler's reagent and Nessler's precipitate. Anal. Chim. Acta., 14, 209-2 2 .

SHafFer F. L., Sprecher J. C., I957. Routine determination of Nitrogen in the microgram Range with Sealed Tube digestion and direct Nesslerization. Anal. Chem., 29, 437-438.

STEIN W.H., Moore S., I 954. The free amino acids of human blood plasma. J. Biol. Chem., 211, 91 5-926.

Thompson J. F., Morrison G. R., i951. Determination of organic nitrogen. Anal. Chent., 23, I I53.

VIGNERON M., I 960 . Determination par titrage potentiométrique de l'azote aminé libre de quelques amino acides, peptides et protéines. Ann. Pharm. frans., 18, 404.

YUEN S. H., PoldARD A. G., I952. The determination of nitrogen in agricultural materials by the NEsSLER Reagent. J.Sci. Food. Agric., 3, $44 \mathrm{I}-447$. 